\title{
论 文
}

\section{基于 AFM 的哺乳动物活细胞成像}

\author{
李密 ${ }^{(1)}$ ，刘连庆 ${ }^{(1)}$ ，席宁 ${ }^{(3)}$ ，王越超 ${ }^{(1)}$ ，董再励 ${ }^{(1)}$ ，肖秀斌 (4)，张伟京 ${ }^{(4)}$ \\ (1) 中国科学院沈阳自动化研究所, 机器人学国家重点实验室, 沈阳 110016; \\ (2) 中国科学院大学, 北京 100049 ; \\ (3) 香港城市大学机械与生物医学工程系, 香港 999077 ; \\ (4) 军事医学科学院附属医院淋巴科, 北京 100071 \\ * 联系人, E-mail: 1qliu@sia.cn; zhangwj3072@163.com \\ 收稿日期: 2013-05-28; 接受日期: 2013-07-04 \\ 国家自然科学基金(批准号: 61175103)和中国科学院、国家外国专家局创新团队国际合作伙伴计划资助项目
}

\begin{abstract}
摘要利用原子力显微镜对活体状态的哺乳动物贴壁细胞和悬浮细胞的形貌结构进行了 成像. 利用时延 AFM 记录了 MCF-7 和 Neuro-2a 细胞在运动过程中细胞超微结构的动态变 化. AFM 连续成像结果直观揭示出在 MCF-7 细胞的收缩过程中会产生很多锯齿状的不断进 行重组的丝状伪足, 且细胞板状伪足的厚度在收缩运动后增加; 而在 Neuro-2a 细胞的伸长 过程中, 细胞骨架从不规则形态重组为伸直的形态. 针对哺乳动物悬浮细胞, 提出了一种结 合聚二甲基硅氧烷微坑机械夹持和多聚赖氨酸静电吸附的细胞固定方法, 并利用该方法对 单个淋巴瘤活细胞进行固定, 实现了对淋巴瘤活细胞表面形貌的 AFM 成像. 实验结果为细 胞运动带来了新的认识, 同时也证明了 PDMS 微坑方法用于哺乳动物悬浮细胞固定的有 效性.
\end{abstract}

关键词

原子力显微镜

细胞运动

板状伪足

细胞骨架

聚二甲基硅氧烷
细胞是生命有机体的基本单位. 在人体内有许 多不同类型的细胞, 每种细胞都有其独特的尺寸、形 状、结构和功能. 近年来的研究表明, 细胞形状在细 胞生长、分化、发育、死亡和肿瘤生长等许多生理过 程中影响细胞的命运 ${ }^{[1,2]}$. 真核细胞及最终由它们所 构成的有机体的形状由周期性的机械感知、机械传导 和机械响应所决定 ${ }^{[3]}$. 细胞形状反映了细胞骨架、细 胞膜及细胞-基底之间的黏附等因素之间的相互作 用 ${ }^{[4]}$. 因此, 研究单个细胞的形状对于揭示生命的奥 秘具有重要意义. 然而, 由于缺乏适合的技术手段, 在很长的时期内都无法对单个活细胞的超微结构进 行观察. 原子力显微镜 (atomic force microscopy, $\mathrm{AFM})^{[5]}$ 的出现为观察活细胞表面超微结构提供了可
能. AFM 利用一根集成到悬臂梁末端的尖锐针尖对 样本进行光栅扫描来获取样本表面形貌. AFM 可以 在溶液环境下工作且具有纳米级的空间分辨率, 这 种独特的优势使得其十分适合于研究活细胞表面纳 米尺度的生理活动 ${ }^{[6]}$. 应用 AFM 研究自然状态下的 生物样本(活细胞、天然态膜蛋白)给细胞生物学带来 了大量新的认识, 包括在纳米尺度理解细胞膜的组 织和动态行为 ${ }^{[7]}$ 、观测细胞器的结构及活动 ${ }^{[8]}$, 以及 解析蛋白质分子的动态结构 ${ }^{[9]}$ 等. 由于 AFM 所具有 的强大功能, AFM 已经成为细胞生物学的重要实验 手段, 是对传统光学显微镜、电子显微镜和 $\mathrm{X}$ 射线结 晶术的重要补充.

迄今为止, AFM 活细胞成像主要集中在哺乳动 
物贴壁细胞和微生物细胞, 对活体状态的哺乳动物 悬浮细胞进行的成像还很少. 这是由于哺乳动物贴 壁细胞和微生物细胞可以很容易地吸附到平坦的基 底. 贴壁细胞可以在基底上延展生长, 因而不需要额 外的处理即可对生长在基底的贴壁细胞进行 AFM 成 像 ${ }^{[10]}$. 对于微生物细胞, 它们尺寸很小且具有坚硬的 细胞壁, 因而可以利用商用化的微孔滤膜 ${ }^{[11]}$ 或多聚 赖氨酸静电吸附法 ${ }^{[12]}$ 对其进行固定后进行 AFM 成像. 哺乳动物悬浮细胞十分柔软, 细胞尺寸大, 且不能在 基底上延展生长, 这种特性使得微孔滤膜法和多聚 赖氨酸静电吸附法均难以对其进行有效固定. Rosenbluth 等人 ${ }^{[13]}$ 提出了基于 MEMS (micro-electromechanical system) 微坑的悬浮细胞固定方法, 但单 纯的微坑机械夹持还不足以对悬浮细胞进行固定(微 坑仅在水平方向对细胞进行固定). 在以前的研究中, 我们针对动物悬浮细胞提出了一种结合 MEMS 微柱 机械夹持和多聚赖氨酸静电吸附的悬浮细胞固定方 法 $^{[14,15]}$ (该方法可在水平方向和垂直方向对细胞进行 固定), 并基于该方法成功实现了对淋巴瘤活细胞表 面形貌的 AFM 成像.

本研究中, 利用时延 AFM 观察了贴壁细胞运动 过程中的动态行为, 并针对哺乳动物悬浮细胞提出 了一种新的细胞固定方法一一结合聚二甲基硅氧烷 (PDMS)微坑机械夹持和多聚赖氨酸静电吸附. 选取 了 2 种贴壁细胞(乳腺癌 MCF-7 细胞系和小鼠神经母 细胞瘤细胞 Neuro- $2 \mathrm{a}$ 细胞系)和 1 种哺乳动物悬浮细 胞 (B 细胞淋巴瘤 Raji 细胞系)用于 AFM 活细胞成像 实验. 对于贴壁细胞, 通过连续获取 AFM 图像观察 了细胞运动过程中细胞超微结构 (板状伪足、细胞骨 架) 的实时变化; 对于悬浮细胞, 利用所提出的细胞 固定方法对其进行固定并在此基础上进行了活细胞 表面形貌的成像, 实验结果证明了所提出的细胞固 定方法的有效性.

\section{1 材料与方法}

\section{1 样本制备}

MCF-7 和 Raji 细胞在 $37^{\circ} \mathrm{C}\left(5 \% \mathrm{CO}_{2}\right)$ 含 $10 \%$ 胎牛 血清的 RPMI-1640 培养液中培养. Neuro-2a 细胞在 $37^{\circ} \mathrm{C}\left(5 \% \mathrm{CO}_{2}\right)$ 含 $10 \%$ 胎牛血清的 DMEM(高糖)培养 液中培养. MCF-7 和 Neuro-2a 细胞在培养血中培养, Raji 细胞在培养瓶中培养. 细胞培养 $24 \mathrm{~h}$ 后用于
AFM 实验. 对于 MCF-7 和 Neuro-2a 细胞, 直接将细 胞培养血从 $\mathrm{CO}_{2}$ 细胞培养箱中取出后置于 $\mathrm{AFM}$ 载物 台上. 对于 Raji 细胞, 使用 PDMS 微坑芯片对其进行 固定. 首先采用氧等离子体(FEMTO, Diener, 德国) 对 PDMS 微坑芯片进行清洗, 然后将多聚赖氨酸溶 液滴至芯片表面, 自然干燥. 以 $1000 \mathrm{r} / \mathrm{min}$ 的速度离 心 5 min 收集 Raji 细胞, 并将细胞滴至覆盖了多聚赖 氨酸的 PDMS 微坑芯片表面. 随后利用双面胶将微 坑芯片粘至载玻片上, 将载玻片置于含 PBS 的培养 皿中, 并将培养血置于 AFM 载物台上.

\subsection{PDMS 微坑}

制作 PDMS 微坑的前提是制作 MEMS 微柱模具. 利用 MEMS 光刻蚀工艺制作微柱模具在参考文 献 ${ }^{[14,15]}$ 中描述. 将液体状态的 PDMS 浇灌到制作好 的微柱模具上, PDMS 即可填充微柱模具上的敞开区 域. 随后将含液体 PDMS 的微柱模具置于烤箱中, $80^{\circ} \mathrm{C}$ 下烘烤 $2 \mathrm{~h}$. 待 PDMS 固化后, 将其从 PDMS 模 具上剥离即得到 PDMS 微坑芯片. 微坑芯片的高度 为 $10 \mu \mathrm{m}$, 直径为 $20 \mu \mathrm{m}$.

\subsection{AFM 成像}

AFM 成像实验在 Dimension 3100 AFM (Bruker, Santa Barbara, CA, 美国)上进行. 采用的 AFM 探针 型号为 MLCT(Bruker, Santa Barbara, CA, 美国). 探 针悬臂梁的弹性系数为 $0.01 \mathrm{~N} / \mathrm{m}$. 悬臂梁的长、宽和 高分别为 310,20 和 $0.55 \mu \mathrm{m}$. 针尖高度为 $2.5 \sim 8.0 \mu \mathrm{m}$, 针尖半径为 $20 \mathrm{~nm}$. 在 AFM 自带的光学显微镜的导 引下, 将 AFM 针尖移动到活细胞表面对细胞表面形 貌进行扫描成像. 对于贴壁细胞(MCF-7 和 Neuro-2a), 成像模式为接触模式. 为了观测细胞板状伪足的运 动, 对细胞局部区域(细胞边缘)进行连续扫描成像, 成像间隔为 $10 \mathrm{~min}$, 成像时长为 $2 \mathrm{~h}$. 对于 Raji 悬浮 细胞, 成像模式为轻敲模式, 驱动频率为 $9.5 \mathrm{kHz}$.

\section{2 结果与讨论}

细胞运动在胚胎发育、伤口愈合、炎症反应和癌 症转移等生理活动中起着重要的作用 ${ }^{[16]}$. 在胚胎发 育过程中, 细胞通过迁移来形成组织、器官和生命有 机体. 在伤口愈合过程中涉及多种细胞的迁移, 而白 细胞则在炎症反应过程中迁移到淋巴结 ${ }^{[17]}$. 在癌症 
的侵袭和转移过程中, 癌细胞在组织内不断运动 ${ }^{[18]}$. 为了迁移, 细胞需要不断修改自身的形状和硬度来 与其周围的组织结构进行反应 ${ }^{[19]}$. 细胞迁移是一个 不断进行协调的过程, 包括肌动蛋白丝的快速变 化 $^{[20]}$, 以及细胞黏附的形成和解离 ${ }^{[21]}$. 近年来在钻 闪㐿引导细胞迁移 ${ }^{[22]}$ 和基质硬度影响细胞分化 ${ }^{[23]}$ 方 面取得了重要进展, 但直接观察细胞迁移过程中细 胞超微结构变化的研究还不多见. 时延 AFM 直接对 活体状态的样本进行纳米精度的观测, 因此为研究 细胞迁移过程中的动态过程提供了有用的工具.

图 1 显示了 MCF-7 细胞收缩过程中的细胞板状 伪足动态变化, 通过将 $\mathrm{MCF}-7$ 培养血从 $\mathrm{CO}_{2}$ 细胞培 养箱中取出并置于 AFM 载物台后, 立即对一个 MCF-7 细胞的边缘部分进行连续 AFM 扫描成像得到. 图 1A $\sim \mathrm{L}$ 是细胞板状伪足在 $2 \mathrm{~h}$ 内连续获取的 $\mathrm{AFM}$ 偏差图. 参考线由图 $1 \mathrm{~A}$ 和 $\mathrm{L}$ 中的虚线指示. 图 $1 \mathrm{~A}$ 是 板状伪足的最初 AFM 图像, 图 $1 \mathrm{~L}$ 是 $2 \mathrm{~h}$ 后的板状伪 足 AFM 图像. 根据参考线, 可以清晰地看到细胞板 状伪足向细胞中央区域收缩. 在图 $1 \mathrm{~A}$ 中, 细胞板状 伪足紧紧地贴附于基底, 且细胞骨架清晰可见. 10 $\min$ 后, 细胞板状伪足开始收缩, 形态也随之发生变 化, 如图 1B 所示. 在图 1C 中, 细胞收缩运动继续进 展, 此时在 AFM 图像上观察不到细胞骨架. $30 \mathrm{~min}$ 后, 可以看到在细胞板状伪足上形成了 4 个小的锯齿状 的丝状伪足, 由图 $1 \mathrm{D}$ 中的圆圈指示. 在图 $1 \mathrm{E}$ 中这 4 个锯齿状的丝状伪足又重组为 3 个丝状伪足, 而在图
$1 \mathrm{~F}$ 中则变成 2 个丝状伪足. 从此时开始, 细胞伪足形 态变化的速度显著降低, 并最终形成一个新的板状 伪足(图 1L). 通过 AFM 图像中的截面曲线测量了细 胞收缩过程中细胞板状伪足厚度的变化. 截面曲线 如图 $1 \mathrm{~A}$ 和 $\mathrm{L}$ 中插图所示. 细胞板状伪足的最初厚度 约为 $100 \mathrm{~nm}$ (图 $1 \mathrm{~A}$ 中的截面曲线计算得到), 而在 2 $\mathrm{h}$ 收缩运动后厚度变为约 $300 \mathrm{~nm}$ (图 $1 \mathrm{~L}$ 中的截面曲 线计算得到). 可以看到细胞板状伪足在收缩运动后 厚度显著增加.

图 2 显示了另外一个 $\mathrm{MCF}-7$ 细胞的 $\mathrm{AFM}$ 连续 扫描图像, 通过将 $\mathrm{MCF}-7$ 培养血从 $\mathrm{CO}_{2}$ 细胞培养箱 中取出并置于 AFM 载物台 $1 \mathrm{~h}$ 后进行扫描成像得到. 图 $2 \mathrm{~A} \sim \mathrm{L}$ 是细胞板状伪足在 $2 \mathrm{~h}$ 内的连续 $\mathrm{AFM}$ 偏差 图. 图 $2 \mathrm{~A}$ 和 $\mathrm{L}$ 中的虚线为参考线. 从图 2 可以看到 细胞并不运动, 且细胞板状伪足的形态也没有变化. $\mathrm{CO}_{2}$ 细胞培养箱内的环境为 $37^{\circ} \mathrm{C}$ 和 $5 \% \mathrm{CO}_{2}$, 而 $\mathrm{CO}_{2}$ 细胞培养箱外的环境为室温大气环境. 当将细胞从 细胞培养箱中取出后, 环境的突然变化可能导致细 胞行为的变化, 这可能使得将细胞从细胞培养箱中 取出后立即进行 AFM 连续成像时可以观测到 MCF-7 细胞的收缩运动. 将细胞置于室温大气环境下 $1 \mathrm{~h}$ 后, 细胞可能已经适应了细胞培养箱外的环境, 因而观 测不到细胞收缩现象. 事实上从图 1 中可以看到细胞 运动在 $30 \mathrm{~min}$ 后便不明显. 研究表明, 细胞在 AFM 实 验中可以存活至少 $3 \mathrm{~h}^{[24,25]}$, 因此在 $3 \mathrm{~h}$ 内进行 AFM 实验以确保细胞仍然是活体状态. 然而, 造成 MCF-7
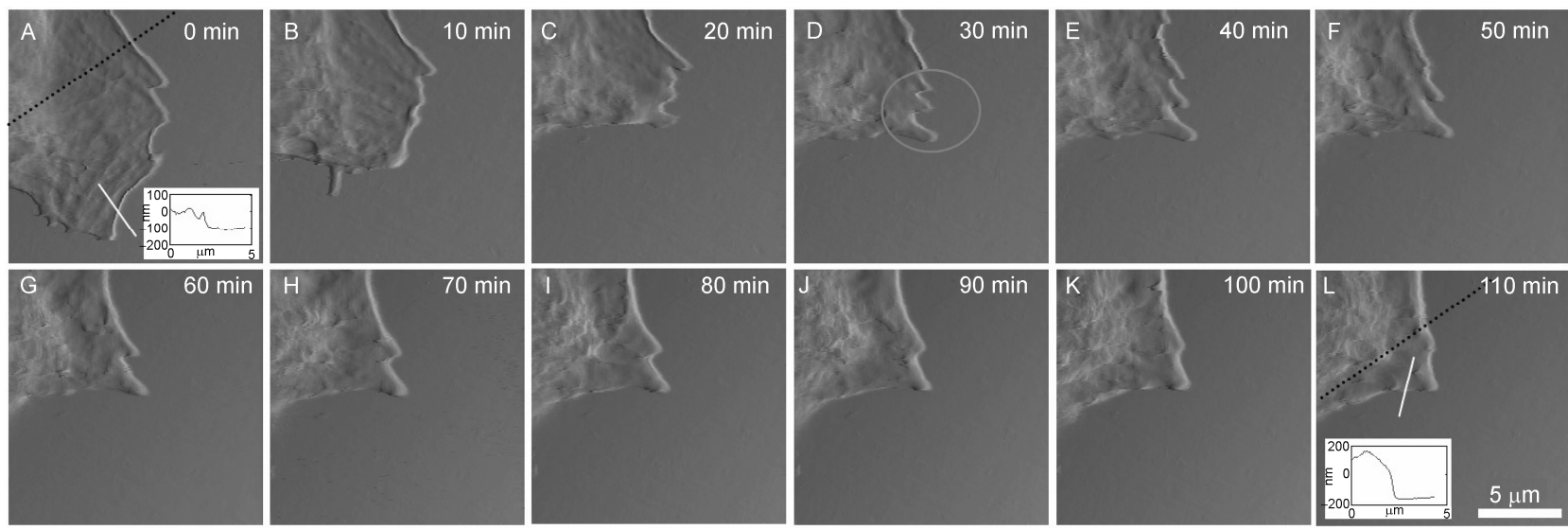

图 1 MCF-7 细胞收缩过程中的细胞板状伪足动态变化

将培养血从 $\mathrm{CO}_{2}$ 细胞培养箱中取出并置于 AFM 载物台后立即对一个 MCF-7 细胞边缘部分进行连续 AFM 扫描成像. 细胞板状伪足向细胞中 心区域收缩, 并出现很多丝状伪足, 由 $\mathrm{D}$ 图中的圆圈所指示. 这些丝状伪足最后重组为一个新的板状伪足 $(\mathrm{L}) . \mathrm{A}$ 和 $\mathrm{L}$ 中的插图为白色线条指 示的 AFM 截面曲线. $\mathrm{A}$ 和 $\mathrm{L}$ 中的虚线为参考线 


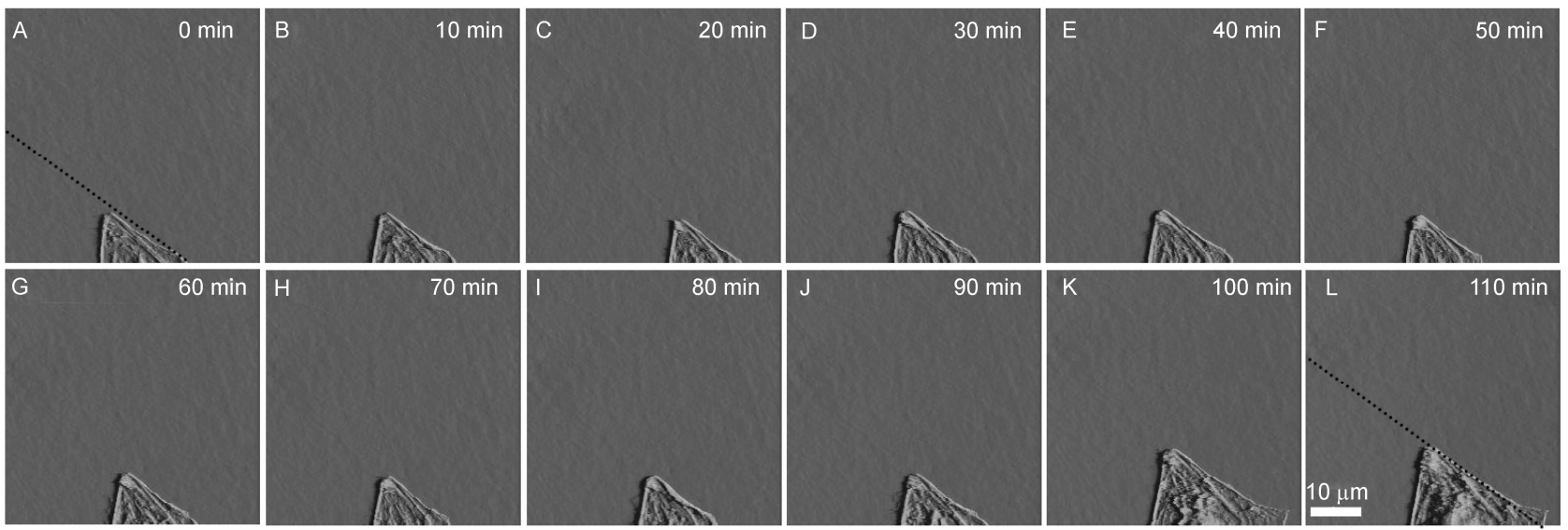

图 2 MCF-7 细胞的连续 AFM 扫描成像

将培养血从 $\mathrm{CO}_{2}$ 细胞培养箱中取出并置于 AFM 载物台 $1 \mathrm{~h}$ 后对另外一个 MCF-7 细胞边缘部分进行连续 AFM 扫描成像. 细胞没有运动. A 和 $\mathrm{L}$ 中的虚线为参考线

细胞收缩运动的具体机制还不清楚, 有待进一步研究.

图 3 显示了 Neuro- $2 \mathrm{a}$ 细胞伸长运动过程中的动 态变化, 通过将培养血从 $\mathrm{CO}_{2}$ 细胞培养箱中取出并 置于 $\mathrm{AFM}$ 载物台 $1 \mathrm{~h}$ 后进行连续 $\mathrm{AFM}$ 成像得到. 图 $3 \mathrm{~A} \sim \mathrm{L}$ 是细胞轴突的连续扫描 AFM 偏差图像. 图 $3 \mathrm{~A}$ 是轴突的最初 AFM 图像, 可以看到此时观察不到细 胞骨架. 图 3A 插图为光学图像, 圆圈指示细胞轴突. 与 MCF-7 细胞的收缩运动相反, Neuro- $2 \mathrm{a}$ 细胞呈现伸 长运动. 随着伸长运动的进展, 在 $\mathrm{AFM}$ 图像中细胞 骨架逐渐清晰可见(图 3F H). 轴突头部的形状不断 变化并最终变为圆形(图 3L); 同时细胞骨架从不规 则形态(图 3I K) 变为伸直的形态(图 3L). 图 4 显示了 另外一个 Neuro- $2 \mathrm{a}$ 细胞的动态变化, 同样是通过将 培养血从 $\mathrm{CO}_{2}$ 细胞培养箱中取出并置于 $\mathrm{AFM}$ 载物台 $1 \mathrm{~h}$ 后进行连续 AFM 成像得到. 可以看到这个 Neuro- $2 \mathrm{a}$ 细胞并不运动, 但是细胞骨架在 $2 \mathrm{~h}$ 内不断 变化. 在最初的 AFM 图像中(图 4A), 细胞骨架不可 见, 但细胞骨架在后续的 AFM 图像中逐渐变得清晰 且细胞骨架变为伸直的形态 (图 4F L). 图 3 和 4 的结 果表明能否利用时延 AFM 观测到 Neuro-2a 细胞的伸 长运动与细胞自身有关, 即同一种类的不同细胞间 有可能具有差异.

比较图 1 4, 可以看到 MCF-7 细胞和 Neuro-2a 细胞具有不同的运动行为. 对于 $\mathrm{MCF}-7$ 细胞, 通过 将细胞培养血从细胞培养箱中取出后立即进行 AFM 连续扫描成像可以观测到细胞的收缩运动, 但将细 胞放置 $1 \mathrm{~h}$ 后便观察不到细胞的收缩运动. 对于 Neuro- $2 \mathrm{a}$ 细胞, 细胞的伸长运动并不具有普遍性, 但
在利用时延 AFM 对其进行连续扫描成像的过程中, 细胞骨架会不断变化. 时延 AFM 是一个用来研究细 胞活动的动态过程的有用工具 ${ }^{[10,26,27]}$. Stadler 等人 ${ }^{[10]}$ 等结合 AFM 和共聚焦激光扫描显微镜(confocal laser scanning microscopy, CLSM)研究了成肌细胞在肌肉生 成过程不同阶段的动态行为, 直观地揭示出在细胞 分裂和融合过程中细胞膜结构的重组. Schoenenberger 和 $\mathrm{Hoh}^{[26]}$ 利用时延 AFM 研究了 MDCK 细胞运 动过程中的细胞动态变化并揭示了细胞超微结构的 动态变化. Ushiki 等人 ${ }^{[27]}$ 应用时延 AFM 研究了人食 管鳞状细胞癌细胞的运动. 本研究中利用时延 AFM 对 MCF-7 细胞和 Neuro-2a 细胞运动过程中的动态过 程进行了连续扫描成像. 实验结果显示, 这 2 种细胞 在处于室温大气环境下时具有不同的运动行为, 且 细胞运动在某种程度上与细胞本身及与在室温大气 环境下放置的时间有关.

通过将贴壁细胞生长在基底上即可容易地对其 进行 AFM 成像. 哺乳动物悬浮细胞不能贴壁延展生 长, 因而需要采用细胞固定方法来将细胞吸附到基 底上. 针对哺乳动物悬浮细胞, 我们提出了一种结合 MEMS 微柱和静电吸附的细胞固定方法 ${ }^{[14,15]}$, 然而 该方法具有价格昂贵、芯片不能重复使用的缺点. 考 虑到 PDMS 价格便宜, 且制作好的微柱模具可以反 复使用. 因此研究了在 MEMS 微柱基础上利用 PDMS 制作微坑的细胞固定方法. 将液体 PDMS 浇灌 到微柱模具上即可制作 PDMS 微坑. 图 5A 是制作好 的 PDMS 微坑实物图. 图 $5 \mathrm{E}$ 是微坑的光学图像. 从 


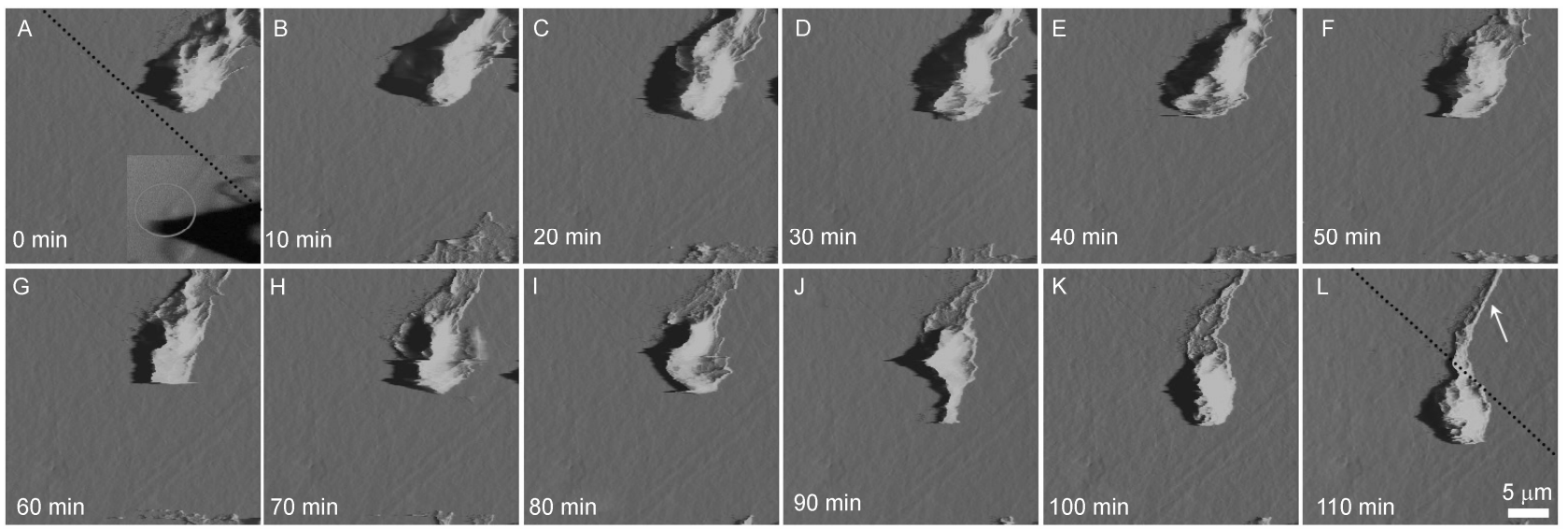

图 3 Neuro-2a 细胞伸长运动过程中的动态变化

将培养血从 $\mathrm{CO}_{2}$ 细胞培养箱中取出并置于 $\mathrm{AFM}$ 载物台 $1 \mathrm{~h}$ 后对一个 Neuro- $2 \mathrm{a}$ 细胞进行连续 $\mathrm{AFM}$ 扫描成像. 细胞轴突在基底上不断伸长, 且 从不规则结构 $(\mathrm{I} \sim \mathrm{K})$ 变为伸直的结构 ( $\mathrm{L}$ 中的箭头指示). $\mathrm{A}$ 和 $\mathrm{L}$ 中的虚线为参考线. $\mathrm{A}$ 中的插图为光学图像

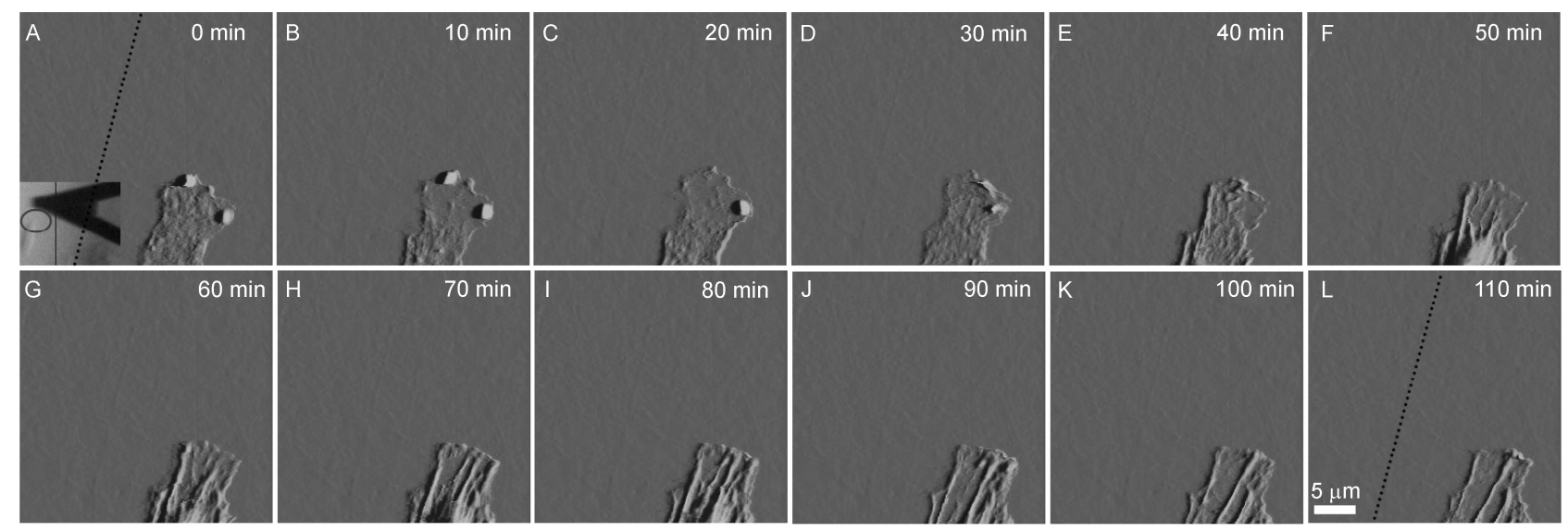

图 4 Neuro-2a 细胞的连续 AFM 扫描成像

将培养血从 $\mathrm{CO}_{2}$ 细胞培养箱中取出并置于 AFM 载物台 $1 \mathrm{~h}$ 后对另外一个 Neuro- $2 \mathrm{a}$ 细胞进行连续 $\mathrm{AFM}$ 扫描成像. 细胞没有运动, 但细胞骨 架不断变化. $\mathrm{A}$ 和 $\mathrm{L}$ 中的虚线为参考线. $\mathrm{A}$ 中的插图为光学图像

光学显微图上可以清晰地看到整齐排列的微坑. PDMS 是一种疏水材料, 在投入使用之前, 需要对微 坑芯片表面进行亲水性处理. 如果不对芯片进行亲 水性处理, 直接将制作的微坑芯片置于 PBS 缓冲液 中, 则利用 AFM 对微坑进行扫描成像(接触模式)时, 发现微坑内会形成一个圆形液滴, 如图 5B 和 C 所示, 分别为 AFM 高度图和偏差图. 从截面曲线图上(图 5D) 可以看到圆形液滴轮廓呈现抛物线的形状. 在实 验中发现, 如果不将微坑内的圆形液滴去除, 在利用 AFM 探针推动细胞至微坑时, 细胞不会掉入坑内. 因此, 为了使细胞能够落入微坑内, 需要对芯片表面 进行改性处理. 利用氧等离子体清洗机对芯片清洗
2 min 后, 将芯片置于 PBS 中, 并利用 AFM 对微坑进 行扫描成像, 成像结果如图 $5 \mathrm{~F}$ 和 $\mathrm{G}$ 所示, 分别为 $\mathrm{AFM}$ 高度图和偏差图. 图 $5 \mathrm{H}$ 为截面曲线图. 从 $\mathrm{AFM}$ 图像上可以看到微坑内的圆形液滴消失. 这是由于 氧等离子清洗改变了 PDMS 微坑芯片的表面特性, 使得其浸润性增强并使得圆形液滴消失.

利用经过氧等离子体改性的 PDMS 微坑芯片并 结合多聚赖氨酸的静电吸附来对淋巴瘤细胞进行固 定并进行轻敲模式AFM 扫描成像, 实验结果如图 6 所示. 微坑可以在水平方向上帮助细胞抵挡探针施 加的扫描力, 而多聚赖氨酸则可以在垂直方向将细 胞吸附到微坑底部. 在光学图像上(图 6E), 可以清晰 

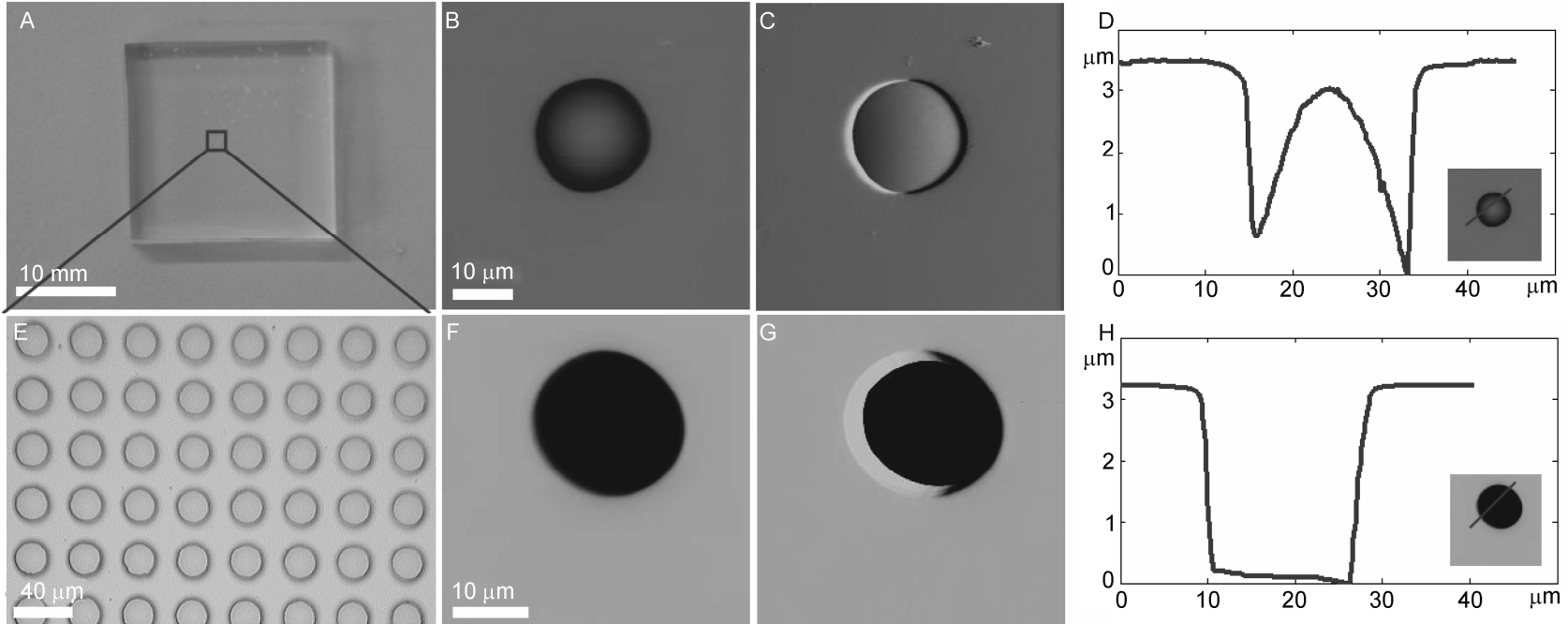

图 5 PDMS 微坑芯片

A: 制作好的微坑芯片实物图. 利用 AFM 在溶液中对氧等离子体清洗前后的微坑进行成像. 未经等离子体清洗的微坑 AFM 高度图(B)、偏差 图(C)和截面曲线图(D). E: 微坑芯片的光学图像. 经过氧等离子体清洗后的微坑 AFM 高度图 $(\mathrm{F})$ 、偏差图 $(\mathrm{G})$ 和截面曲线图 $(\mathrm{H})$
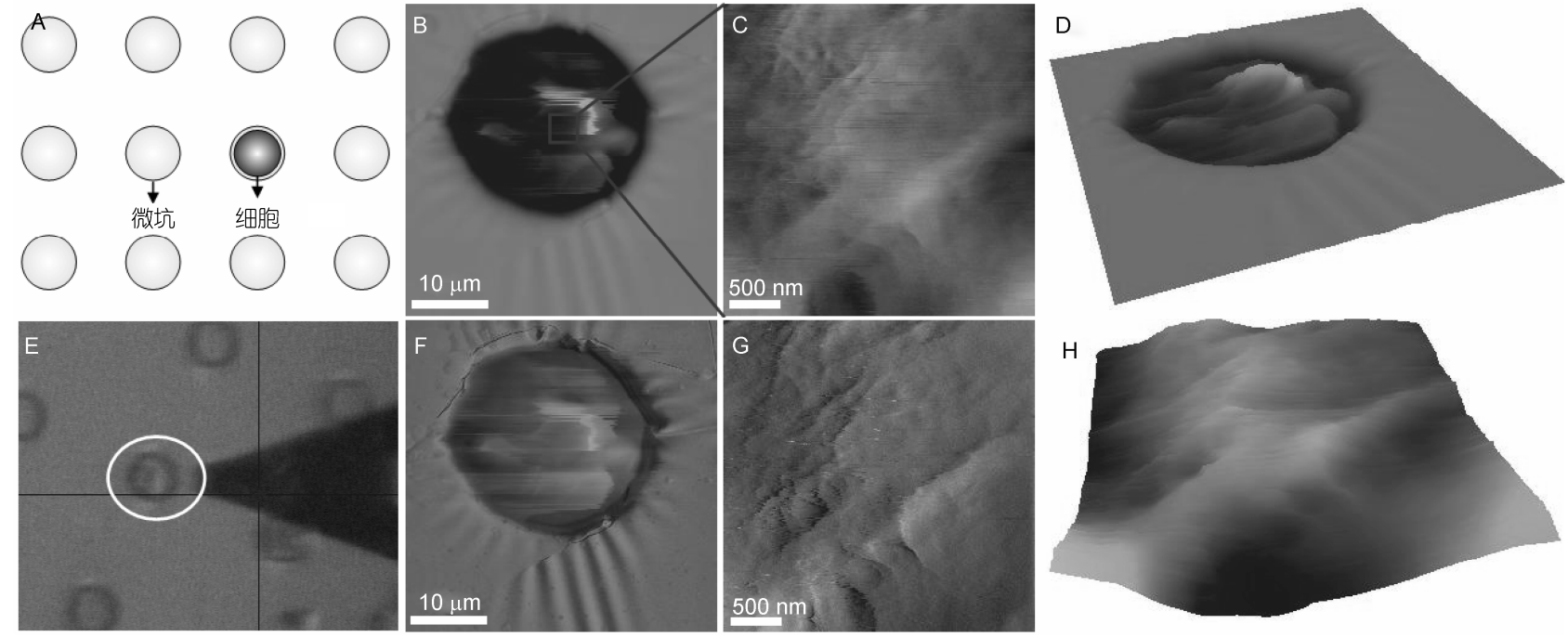

图 6 基于 PDMS 微坑固定的活体状态悬浮细胞成像

利用 PDMS 微坑夹持和多聚赖氨酸静电吸附相结合的细胞固定方法对淋巴瘤活细胞进行固定, 并在此基础上进行轻敲模式 AFM 成像. A: PDMS 微坑固定原理图; E: 在光学显微镜的导引下将 AFM 探针移动到被微坑夹持的细胞表面. 细胞整体高度图(B)、相位图(F)和三维图(D). 细胞局部区域的相位图 $(\mathrm{C})$ 、振幅图 $(\mathrm{G})$ 和三维图 $(\mathrm{H})$

地观察到被微坑固定的细胞(图 6E 中的圆圈指示), 随后移动 AFM 针尖到细胞表面对细胞进行成像. 图 $6 \mathrm{~B}, \mathrm{D}$ 和 $\mathrm{F}$ 分别为细胞整体的高度图、三维图和相位 图. 图 $6 \mathrm{C}, \mathrm{G}$ 和 $\mathrm{H}$ 分别为细胞表面局部区域的相位 图、振幅图和三维图. 从细胞局部图像上可看到细胞 表面粗糙、起伏不平. 如果不在微坑芯片上覆盖一层
多聚赖氨酸，则在利用 AFM 对细胞进行成像时细胞 会在坑内不断移动. 在以前的研究中, 利用硅材料微 柱芯片来固定活体状态的淋巴瘤细胞 ${ }^{[14,15]}$, 细胞可 以牢固地吸附到覆盖了多聚赖氨酸的芯片基底. 比 较微坑固定方法获取的 AFM 图像和微柱固定方法获 取的 AFM 图像，基于微坑固定获取的细胞整体图质 
量不如基于微柱固定方法获取的细胞整体图像. 这 可能与材料的差异有关. 硅材料为亲水性, 而 PDMS 为疏水性. 尽管利用氧等离子体清洗可改善 PDMS 的亲水性能, 但改性后的 PDMS 与硅的亲水性仍可 能有差异. 此外, 硅材料十分坚硬, 而 PDMS 很柔软, 这种硬度差异也可能导致对细胞吸附能力的不同. 对细胞表面局部区域获取图像时(图 6C, G 和 $\mathrm{H}$ ), 图 像质量提高. 这是由于随着扫描范围的缩小, 细胞膜 的运动对成像质量的影响降低, 成像质量提高. Dague 等人 ${ }^{[28]}$ 利用加工出的 PDMS 微坑对酿酒酵母 (Saccharomyces cerevisiae) 和烟曲酶真菌狍子(Aspergillus fumigatus)进行机械固定并在此基础上实现了对 酵母菌和孢子的 AFM 成像. 微生物细胞具有坚硬的 细胞壁, 因而很容易获得高质量 AFM 图像. 本研究 中利用 PDMS 微坑来固定哺乳动物悬浮细胞. 哺乳 动物细胞十分柔软, 尺度大, 难以获得高质量图像. 目前利用 AFM 对活体状态的哺乳动物细胞成像的分
辨率限制在 $50 \mathrm{~nm}^{[6]}$, 这是由于细胞膜的动态柔软特 性使得扫描针尖很容易导致细胞膜的形变 ${ }^{[29]}$, 影响 到成像分辨率. 为了提高 AFM 活细胞成像的分辨率, 需要研究能够对细胞膜运动进行有效抑制的方法, 如含有小孔的薄膜 ${ }^{[30]}$.

\section{3 结论}

$\mathrm{AFM}$ 为高分辨率观测活细胞表面的生理活动提 供了新的工具 ${ }^{[31 ~ 34]}$. 利用时延 AFM 直接观察了贴壁 细胞运动过程中细胞超微结构 (板状伪足、细胞骨架) 的实时变化, 为细胞运动提供了新的认识. 同时针对 哺乳动物悬浮细胞, 提出了一种结合 PDMS 微坑和 静电吸附的细胞固定方法, 并基于该方法对活体状 态的淋巴瘤细胞的固定, 实现了对细胞表面形貌的 AFM 成像, 实验结果证明了该方法的有效性, 为进 一步研究单个活体状态哺乳动物悬浮细胞的生理活 动奠定了基础.

\section{参考文献}

1 Mogilner A, Keren K. The shape of motile cells. Curr Biol, 2009, 19: R762-R771

2 Kilian K A, Bugarija B, Lahn B T, et al. Geometric cues for directing the differentiation of mesenchymal stem cells. Proc Natl Acad Sci USA, 2010, 107: 4872-4877

3 Vogel V, Sheetz M. Local force and geometry sensing regulate cell functions. Nat Rev Mol Cell Biol, 2006, 7: 265-275

4 Keren K, Pincus Z, Allen G M, et al. Mechanism of shape determination in motile cells. Nature, 2008, 453: 475-480

5 Binnig G, Quate C F, Gerber C. Atomic force microscope. Phys Rev Lett, 1986, 56: 930-933

6 Muller D J, Dufrene Y F. Atomic force microscopy: a nanoscopic window on the cell surface. Trends Cell Biol, 2011, 21: 461-469

7 Heinisch J J, Lipke P N, Beaussart A, et al. Atomic force microscopy-looking at mechanosensors on the cell surface. J Cell Sci, 2012, 125: 4189-4195

8 Schafer C, Shahin V, Albermann L, et al. Aldosterone signaling pathway across the nuclear envelope. Proc Natl Acad Sci USA, 2002, 99: 7154-7159

9 Mari S A, Pessoa J, Altieri S, et al. Gating of the Mlotik1 potassium channel involves large rearrangements of the cyclic nucleotide-binding domains. Proc Natl Acad Sci USA, 2011, 108: 20802-20807

10 Stadler B, Blattler T M, Franco-Obregon A. Time-lapse imaging of in vitro myogenesis using atomic force microscopy. J Microsc, 2010, 237: 63-69

11 Alsteens D, Dupres V, Yunus S, et al. High-resolution imaging of chemical and biological sites on living cells using peak force tapping atomic force microscopy. Langmuir, 2012, 28: 16738-16744

12 Fatner G E, Barbero R J, Gray D S, et al. Kinetics of antimicrobial peptide activity measured on individual bacterial cells using high-speed atomic force microscopy. Nat Nanotechnol, 2010, 5: 280-285

13 Rosenbluth M J, Lam W A, Fletcher D A. Force microscopy of nonadherent cells: a comparison of leukemia cell deformability. Biophys J, 2006, 90: 2994-3003

$14 \mathrm{Li} \mathrm{M}$, Liu L, Xi N, et al. Imaging and measuring the rituximab-induced changes of mechanical properties in B-lymphoma cells using atomic force 
microscopy. Biochem Biophys Res Commun, 2011, 404: 689-694

15 Li M, Liu L, Xi N, et al. Drug-induced changes of topography and elasticity in living B lymphoma cells based on atomic force microscopy. Acta Phys Chim Sin, 2012, 28: 1502-1508

16 Bershadsky A D, Kozlov M M. Crawling cell locomotion revisited. Proc Natl Acad Sci USA, 2011, 108: 20275-20276

17 Franz C M, Jones G E, Ridley A J. Cell migration in development and disease. Dev Cell, 2002, 2: 153-158

18 Yamazaki D, Kurisu S, Takenawa T. Regulation of cancer cell motility through actin reorganization. Cancer Sci, 2005, 96: 379-386

19 Friedl P, Wolf K. Tumor-cell invasion and migration: diversity and escape mechanisms. Nat Rev Cancer, 2003, 3: 362-374

20 Pollard T D, Cooper J A. Actin, a central player in cell shape and movement. Science, 2009, 326: 1208-1212

21 Mitra S K, Hanson D A, Schlaepfer D D. Focal adhesion kinase: in command and control of cell motility. Nat Rev Mol Cell Biol, 2005, 6: 56-68

22 Wei C, Wang X, Chen M, et al. Calcium flickers steer cell migration. Nature, 2009, 457: 901-905

23 Engler A J, Sen S, Sweeney H L, et al. Matrix elasticity directs stem cell lineage specification. Cell, 2006, 126: 677-689

24 Schaus S S, Henderson E R. Cell viability and probe-cell membrane interactions of XR1 glial cells imaged by atomic force microscopy. Biophys J, 1997, 73: 1205-1214

25 Li Y, Zhang J, Zhang B. Atomic force microscopy study on chlorpromazine-induced morphological changes of living HeLa cells in vitro. Scanning, 2009, 31, 259-265

26 Schoenenberger C A, Hoh J H. Slow cellular dynamics in MDCK and R5 cells monitored by time-lapse atomic force microscopy. Biophys J, 1994, 67: 929-936

27 Ushiki T, Hitomi J, Umemoto T, et al. Imaging of living cultured cells of an epithelial nature by atomic force microscopy. Arch Histol Cytol, 1999, 62: $47-55$

28 Dague E, Jauvert E, Laplatine L, et al. Assembly of live micro-organisms on microstructured PDMS stamps by convective/capillary deposition for AFM bio-experiments. Nanotechnology, 2011, 22: 395102

29 Muller D J, Dufrene Y F. Force nanoscopy of living cells. Curr Biol, 2011, 21: R212-R216

30 Ando T. High-speed atomic force microscopy coming of age. Nanotechnology, 2012, 23: 062001

31 李密, 刘连庆, 席宁, 等. 基于 AFM 单分子力谱技术的 CD20-Rituximab 间相互作用力测量. 科学通报, 2011, 56: 2681-2688

32 李密, 刘连庆, 席宁, 等. 基于 AFM 的红细胞及不同侵袭程度癌细胞的成像及机械特性测量. 中国科学：生命科学, 2012, 42: 919-925

33 李密, 刘连庆, 席宁, 等. AFM 单细胞单分子形貌成像的研究进展. 科学通报, 2013, 58: 1711-1718

34 Li M, Liu L Q, Xi N, et al. Mapping CD20 molecules on the lymphoma cell surface using atomic force microscopy. Chin Sci Bull, 2013, 58: 1516-1519 